\title{
Custo real do tratamento do glaucoma para o paciente
}

\author{
The real cost of glaucoma treatment for an outpatient
}

\author{
Larissa Pedroso ${ }^{(1)}$ \\ Edenilson dos Santos Carvalho Junior ${ }^{(2)}$ \\ Auguusto Paranhos Júnior ${ }^{(3)}$ \\ João Antônio Prata Júnior ${ }^{(4)}$ \\ Paulo Augiusto de Arruda Mello ${ }^{(5)}$
}

\begin{abstract}
RESUMO
Objetivo: Avaliar o custo do tratamento do glaucoma para o paciente, numa instituição de ensino médico.

Casuística e Métodos: Foram aplicados, aleatoriamente, questionários, na forma de entrevista ativa, a 94 pacientes do ambulatório do Setor de Glaucoma do Departamento de Oftalmologia da Universidade Federal de São Paulo, analisando gastos pessoais e com medicamentos antiglaucomatosos. Os dados foram agrupados, tabulados e foi realizada separação da amostra em dois grupos: pacientes cirúrgicos e nãocirúrgicos.

Resultados: A mediana do gasto total anual estimado com glaucoma foi de $R \$ 480,15$, o equivalente a $13 \%$ da renda anual mediana dos pacientes. Separadamente, o grupo dos pacientes cirúrgicos obteve uma mediana dos

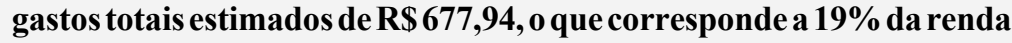
mediana anual. Já o grupo dos pacientes não-cirúrgicos, apresentou mediana de $\mathbf{R} \$ 393,54$, representando $11 \%$ da renda mediana anual. Foi encontrada diferença estatisticamente significante entre os grupos cirúrgico e não-cirúrgico para o gasto total anual $(\mathbf{p}=0,012)$.

Conclusão: Observou-se que o custo do tratamento na renda familiar de pessoas portadoras de glaucoma é considerável, mesmo em uma instituição pública
\end{abstract}

Palavras-chave: Glaucoma, custo, tratamento.

Apresentado sob a forma de tema livre no XIII Congresso Brasileiro de Prevenção da Cegueira e Reabilitação Visual em 07 de setembro de1998 no Hotel Glória, Rio de Janeiro -RJ.

Apoio: bolsa de iniciação científica do $\mathrm{CNPq}$ Os autores declaram não possuir interesse financeiro no marketing dos medicamentos mencionados no estudo.

(1) Residente de Oftalmologia da Escola Paulista de Medicina / Universidade Federal de São Paulo Departamento de Oftalmologia;

(2) Aluno do $5^{\circ}$ ano médico da Escola Paulista de Medicina / Universidade Federal de São Paulo;

(3) Pós-graduando da Escola Paulista de Medicina Universidade Federal de São Paulo - Departamento de Oftalmologia - Setor de Glaucoma;

(4) Médico, Doutor, Escola Paulista de Medicina / Universidade Federal de São Paulo - Departamento de Oftalmologia / Chefe do Setor de Glaucoma;

(5) Professor adjunto da Escola Paulista de Medicina / Universidade Federal de São Paulo - Departamento de Oftalmologia. - Setor de Glaucoma

Endereço para correspondência: $\mathrm{Dr}^{\mathrm{a}}$ Larissa Pedroso - Rua João Julião, 296 - Ap. 64-B. Bela Vista São Paulo (SP). CEP 01323-020. Fone: 289-0707. e-mail: larrisap@oftalmo.epm.br

\section{INTRODUÇÃO}

O glaucoma é um problema de saúde pública, e uma das mais importantes causas de cegueira no Brasil e no mundo. Nos Estados Unidos, 2,25 milhões de pessoas acima de 45 anos têm glaucoma ${ }^{1}$. Segundo a OMS, a incidência de glaucoma no mundo é estimada em 2,4 milhões de casos por ano, a prevalência em 6,2 milhões de casos e a prevalência de cegueira por glaucoma crônico simples, em 5,2 milhões de pessoas (dado relativo ao ano de 1997).

Aproximadamente, $10 \%$ dos pacientes já se apresentam cegos na primeira consulta ${ }^{2}$, dessa forma Jones e col. sugerem um "screening" populacional para a detecção da doença precocemente ${ }^{3}$. O tratamento pode prevenir ou retardar o aparecimento da cegueira, com melhora da qualidade de vida, e evitando despesas com a cegueira. Os Estados Unidos gastam pelo menos US\$ 94 milhões por ano no tratamento do glaucoma ${ }^{4}$, sendo que no ano de 1992, foram gastos US\$ 2,2 bilhões no tratamento e na prevenção da cegueira causada por glaucoma ${ }^{1}$.

Estudos sobre o custo diário do tratamento do glaucoma com B-bloqueadores mostram que existem várias diferenças no custo de colírio para
} 
colírio e de farmácia para farmácia ${ }^{5-7}$. O tratamento cirúrgico precoce com trabeculectomia se mostrou menos oneroso quando comparado ao tratamento clínico exclusivamente ${ }^{8}$.

Campanhas de triagem para detecção de glaucoma, baseadas na pressão intra-ocular foram realizadas no Brasil, entretanto a relação custo por casos detectados e tratados não foi avaliada ${ }^{9-11}$.Tais custos devem ser factíveis para o planejamento do sistema público hospitalar ${ }^{3}$.

Há escassez de estudos sobre custos de tratamentos em oftalmologia no Brasil, especialmente sobre o tratamento do glaucoma. Este estudo pretende avaliar o custo do tratamento do glaucoma para o paciente, numa instituição de ensino médico.

\section{CASUÍSTICA E MÉTODOS}

Para atender aos objetivos estabelecidos, definiu-se uma amostra de 94 pacientes do ambulatório do Setor de Glaucoma do Departamento de Oftalmologia da Escola Paulista de Medicina - Universidade Federal de São Paulo (EPM/ UNIFESP). Os pacientes, selecionados de forma aleatória, foram submetidos a um questionário (Quadro 1), na forma de entrevista ativa, na sala de espera do ambulatório, no período compreendido entre 16 de abril e 14 de maio de 1997.

Foram formuladas questões referentes a sexo, idade, estado civil, custo do deslocamento dos pacientes e seus acompanhantes desde o seu domicílio até o ambulatório do Setor de Glaucoma do Departamento de Oftalmologia da EPM/ UNIFESP e das ausências no trabalho (paciente e acompanhante). Foram também colhidas informações gerais sobre renda familiar, gastos com alimentação (paciente e acompanhante), grau de instrução, número de visitas ambulatoriais nos últimos doze meses e o tempo diário gasto por cada paciente, em dia de consulta. Este tempo corresponde àquele gasto pelo paciente desde a saída de seu domicílio até seu retorno ao mesmo (transporte, espera no ambulatório, etc.). Por fim, identificaram-se as medicações mais utilizadas e as suas prescrições mensais para cada paciente (Quadro 1).

O preço dos medicamentos foi estimado por uma média realizada entre três farmácias localizadas em pontos diferentes da cidade.

Para as análises estatísticas, a amostra foi separada em dois grupos: pacientes cirúrgicos $(\mathrm{CG})$ e não cirúrgicos $(\mathrm{NC})$. Os cirúrgicos, pacientes em pós-operatório de cirurgia antiglaucomatosa de no máximo um ano da data de sua entrevista, uma vez que o questionário se referia aos últimos doze meses.

Para a amostra de pacientes pesquisados, independentemente do grupo em que foi enquadrado, adotou-se o seguinte procedimento para montagem da base de cálculo do custo real do tratamento do glaucoma:

1) Para cada paciente e seu respectivo acompanhante fo-

Quadro1. Modelo do questionário utilizado na entrevista aos pacientes do ambulatório do Setor de Glaucoma do Departamento de Oftalmologia da EPM/UNIFESP.

\begin{tabular}{|c|c|c|c|c|c|c|c|c|}
\hline \multicolumn{4}{|l|}{ Nome } & \multicolumn{5}{|l|}{ Sexo: } \\
\hline \multicolumn{3}{|c|}{ Grau Instrução: } & \multicolumn{3}{|c|}{ Idade: } & \multicolumn{3}{|c|}{ Estado Civil: } \\
\hline \multicolumn{3}{|c|}{ Cirurgia antiglaucomatosa } & & & & Sim & (há quanto temp & \\
\hline \multicolumn{5}{|c|}{ Transporte } & \multicolumn{4}{|c|}{ Falta ao serviço } \\
\hline Paciente: & Número/Custo & Acomp & & $\mathrm{o} /$ Custo & Paciente & Custo & Acompanhante & Custo \\
\hline Ônibus & & \multicolumn{3}{|l|}{ Ônibus } & \multicolumn{4}{|c|}{ Números de visitas nos últimos 12 meses } \\
\hline Metrô & & \multicolumn{2}{|l|}{ Metrô } & & \multicolumn{4}{|c|}{ Tempo Gasto por Visita } \\
\hline Táxi & & \multicolumn{2}{|l|}{ Táxi } & & \multicolumn{4}{|c|}{ Gasto com Alimentação } \\
\hline Carro & & \multicolumn{2}{|l|}{ Carro } & & \multicolumn{4}{|c|}{ Renda Familiar Mensal } \\
\hline Lotação & & \multicolumn{2}{|l|}{ Lotação } & & \multicolumn{2}{|c|}{ Medicamento } & \multicolumn{2}{|l|}{ Frascos por mês } \\
\hline Trem & & \multicolumn{2}{|l|}{ Trem } & & & & & \\
\hline À pé & & \multicolumn{2}{|l|}{ À pé } & & & & & \\
\hline
\end{tabular}


ram tabulados os valores totais correspondentes aos itens transporte, alimentação e ausência ao trabalho, despendidos em um dia de visita ao ambulatório, multiplicados pelo número de visitas em um período de doze meses; para o medicamento, adotou-se o critério de multiplicar o preço do frasco pelo número de frascos utilizados no período de doze meses. A soma destes valores corresponde ao gasto total anual para cada paciente;

2) Outra investigação foi separar os gastos totais por cada item referenciado para cada paciente. Dessa forma, obteve-se os gastos com transporte, alimentação, medicamentos e ausência ao trabalho separadamente no mesmo período de doze meses;

Organizadas tabelas com os dados acima, foram obtidos os valores de "custo total" e valores de "custo para cada item".

$\mathrm{O}$ custo do transporte foi calculado com base no preço vigente no período da pesquisa: ônibus $R \$ 0,85$, metrô e trem $R \$$ 1,00 cada. Quanto ao táxi e veículo próprio, os valores dos gastos estimados eram fornecidos pelo próprio paciente. Pacientes aposentados são isentos das tarifas de transportes públicos, sendo esta informação levada em consideração para os cálculos.

Para efeito de cálculo dos gastos totais e discriminados sobre a renda familiar anual adotou-se o valor de mediana, em função da discrepância dos valores obtidos na pesquisa. $O$ salário mínimo vigente na época da pesquisa era de $\mathrm{R} \$ 112,00$.

A análise estatística se deu por meio de teste de análise de variância por posto de Kruskal-Wallis, soma de postos de Mann-Whitney e correlação de Spearmann. Foi considerado risco alfa de 0,05 .

\section{RESULTADOS}

A mediana do gasto total anual estimado com glaucoma foi de $\mathrm{R} \$ 480,15$, o equivalente a $13 \%$ da renda anual mediana dos pacientes. Quando estudados os grupos CG e NC separadamente, o primeiro obteve uma mediana dos gastos totais estimados de $\mathrm{R} \$ 677,94$, o que corresponde a $19 \%$ da renda mediana anual, enquanto o segundo, mediana de $\mathrm{R} \$ 393,54$, representando $11 \%$ da renda mediana anual (Tabela 1). O teste de Mann-Whitney mostrou que as diferenças foram estatisticamente significantes $(p=0,012)$.

A amostra constou de 94 pacientes sendo 46 homens e 48 mulheres. A renda familiar mensal mediana foi de $\mathrm{R} \$ 300,00$ (Tabela 2). Em sua maioria são pacientes aposentados $(48,94 \%)$, sendo que $11,70 \%$ trabalham e $39,36 \%$ não trabalham e não são aposentados. Quanto ao sexo foi detectada diferença estatística nos gastos totais estimados apenas grupo $\mathrm{NC}(\mathrm{p}=0,038)$, no qual as mulheres apresentaram maiores gastos.

No grupo CG, 42,11\% dos pacientes compareceram ao ambulatório entre 21 e 30 vezes em 12 meses. Já entre no grupo $\mathrm{NC}, 42,67 \%$ visitaram o ambulatório até 10 vezes no mesmo período (Tabela 3 ).

Estatisticamente foi encontrada diferença significante entre os grupos $\mathrm{CG}$ e $\mathrm{NC}$ quando comparados o gasto total estimado ( $\mathrm{p}=$ $0,012)$, o gasto anual com transportes $(p=0,029)$, o tempo gasto por visita $(\mathrm{p}=0,019)$ e o número de visitas por ano $(\mathrm{p}=0,045)$. Quanto ao gasto anual com colírios não foi encontrada diferença estatística. A tabela 4 mostra estes valores acompanhados das respectivas medianas.

A faixa etária dos entrevistados é predominantemente acima de 50 anos $(70,21 \%)$. Não foi encontrada correlação significativa entre idade e gastos totais e descriminados para os grupos $\mathrm{CG}$ e NC.

A amostra é formada basicamente por indivíduos com $1^{\circ}$ grau incompleto (Gráfico 1). Não foi detectada diferença estatisticamente significante para o grau de instrução quanto aos gastos com colírio, gastos totais para os grupos CG e NC (para $\mathrm{p}<0,05)$, mas encontrou-se diferença estatística para o grupo $\mathrm{CG}$ quando comparada a renda com o grau de instrução $(\mathrm{p}=$ 0,014).

Pode-se observar que a amostra é formada basicamente de indivíduos com renda familiar mensal média inferior a 3 salário mínimos (Gráfico 2). Os valores de renda familiar apresentaram grande discrepância, o que levou a adoção do valor da mediana para efeito de cálculos (Tabela 2). Não foi encontrada correlação significativa entre renda e gastos totais e descriminados para os grupos CG e NC.

\section{DISCUSSÃO}

Avaliando o poder aquisitivo da amostra, vimos que a renda familiar mediana é próxima de três salários mínimos para os grupos $\mathrm{CG}$ e $\mathrm{NC}$ e que a mediana do gasto com glaucoma

\begin{tabular}{|c|c|c|c|}
\hline \multicolumn{4}{|c|}{ Gasto Anual Total Estimado } \\
\hline Variável & Amostra Total & Grupo Cirúrgico & Grupo Não Cirúrgico \\
\hline $\mathrm{n}$ & 94 & 19 & 75 \\
\hline Média $\pm \mathrm{DP}^{*}$ & $627,00 \pm 597,10$ & $806,26 \pm 598,28$ & $581,59 \pm 592,17$ \\
\hline Mediana* & 480,15 & 677,94 & 393,54 \\
\hline Variação* & 7,20 a 2698,68 & 161,10 a 2457,54 & 7,20 a 2698,68 \\
\hline$\%$ sobre renda anual ${ }^{\star \star}$ & 13 & 19 & 11 \\
\hline
\end{tabular}




\begin{tabular}{|c|c|c|c|c|}
\hline \multicolumn{5}{|c|}{ Estatística Descritiva } \\
\hline \multicolumn{5}{|c|}{ Amostra total $(n=94)$} \\
\hline & Média & DP & Mediana & Variação \\
\hline Idade (anos) & 55,20 & 21,57 & 61 & 0,6 a 90 \\
\hline Visitas ao Oftalmologista/ano & 17,45 & 13,78 & 12 & 1 a 60 \\
\hline Tempo por visita (horas) & 7,02 & 3,78 & 6 & 2 a 28 \\
\hline Renda familiar mensal $(R \$)$ & 390,61 & 306,61 & 300,00 & 112,00 a $2.100,00$ \\
\hline \multicolumn{5}{|c|}{ Grupo Cirúrgico $(n=19)$} \\
\hline & Média & DP & Mediana & Variação \\
\hline Idade (anos) & 55,58 & 22,97 & 66 & 7 a 84 \\
\hline Visitas ao Oftalmologista/ano & 21,79 & 13,31 & 24 & 4 a 48 \\
\hline Tempo por visita (horas) & 8,18 & 4,28 & 7 & 4 a 24 \\
\hline Renda familiar mensal $(\mathrm{R} \$)$ & 428,89 & 457,59 & 300,00 & 112,00 a $2.100,00$ \\
\hline \multicolumn{5}{|c|}{ Grupo Não Cirúrgico $(n=75)$} \\
\hline & Média & DP & Mediana & Variação \\
\hline Idade (anos) & 55,11 & 21,36 & 60 & 0,6 a 90 \\
\hline Visitas ao Oftalmologista/ano & 16,35 & 13,77 & 12 & 1 a 60 \\
\hline Tempo por visita (horas) & 6,72 & 3,62 & 6 & 2 a 28 \\
\hline Renda familiar mensal ( $R \$)$ & 380,91 & 258,34 & 300,00 & 112,00 a $1.500,00$ \\
\hline
\end{tabular}

equivale a $13 \%$ desta renda. Entretanto analisando-se separadamente os grupos obtêm-se um consumo de $19 \%$ da renda para o grupo CG e de $11 \%$, para o grupo NC (Tabela 1). Isto era esperado uma vez que os pacientes cirúrgicos necessitam de um acompanhamento intenso dos níveis de pressão intra-ocular e aspecto cirúrgico, além de consumir uma maior variedade e quantidade de medicamentos durante o período pós-operatório, e principalmente no pós-operatório recente.

$\mathrm{O}$ gasto total estimado para um ano de tratamento para o grupo CG mostra uma tendência a gastos maiores, em virtude dos fatores expostos anteriormente. Observou-se diferença estatisticamente significante entre os grupos CG e NC nesse sentido $(p=0,012)$.

Foi encontrada diferença estatisticamente significante quanto ao sexo comparado ao gasto total apenas no grupo $\mathrm{NC}$, no qual foi encontrado um gasto maior para o sexo feminino. Este achado não era o esperado, pois não existem relatos na literatura a respeito de diferenças entre as incidências, quanto a evolução clínica e quanto ao tratamento do glaucoma entre os sexos. Entretanto, Kobelt-Nguyen e col. também encontraram gastos maiores entre as mulheres nos EUA, e consideraram como um artefato estatístico ${ }^{12}$.

O grupo $\mathrm{CG}$ obteve maior número de visitas que o grupo

\begin{tabular}{|c|c|c|}
\hline Visitas/ano & Grupo cirúrgico & Grupo não cirúrgico \\
\hline 1 a 10 & $21,05 \%$ & $42,67 \%$ \\
\hline 11 a 20 & $21,05 \%$ & $20,00 \%$ \\
\hline 21 a 30 & $42,11 \%$ & $24,00 \%$ \\
\hline 31 ou mais & $17,79 \%$ & $13,33 \%$ \\
\hline
\end{tabular}

NC (Tabela 2 e 3), pois que estando em pós-operatório (período de um ano após a cirurgia) necessitam de acompanhamento mais rigoroso em relação aos níveis de pressão intraocular e aspecto cirúrgico, principalmente no pós-operatório imediato.

Quanto ao tempo de cada visita, o grupo CG também apresentou maior tempo de visita em relação ao grupo NC (Tabela 2). É importante lembrar que o tempo gasto por visita se deve a consulta e principalmente a espera no departamento e não ao deslocamento até o mesmo e que este tempo representa, para ambos os grupos, a dedicação de um dia inteiro para o tratamento. Foi encontrada diferença estatisticamente significante entre os grupos $\mathrm{CG}$ e $\mathrm{NC}$ tanto para número de visitas quanto para tempo gasto por visita (Tabela 4).

Um fato possível é o de o paciente com melhor instrução ou com melhor renda ser alvo de tratamentos mais onerosos, fato não encontrado em nossa amostra. Não observou-se diferença estatisticamente significante nos grupos CG e NC quanto a grau de instrução com gasto total ou com gasto com medicamentos.

\begin{tabular}{|c|c|c|c|}
\hline \multicolumn{4}{|c|}{ Soma de postos de Mann-Whitney } \\
\hline Variável & $\begin{array}{c}\text { Grupo } \\
\text { Cirúrgico }\end{array}$ & $\begin{array}{c}\text { Grupo Não } \\
\text { Cirúrgico }\end{array}$ & Valor de $p$ \\
\hline Gasto Colírio (R\$) & 517,14 & 249,12 & NS $(p=0,102)$ \\
\hline Gasto Transporte $(\mathrm{R} \$)$ & 68,40 & 14,80 & $S(p=0,026)$ \\
\hline Gasto Total $(\mathrm{R} \$)$ & 677,95 & 393,54 & $S(p=0,012)$ \\
\hline Tempo por visita (horas) & 7 & 6 & $S(p=0,020)$ \\
\hline Número de visitas & 24 & 12 & $S(p=0,045)$ \\
\hline
\end{tabular}




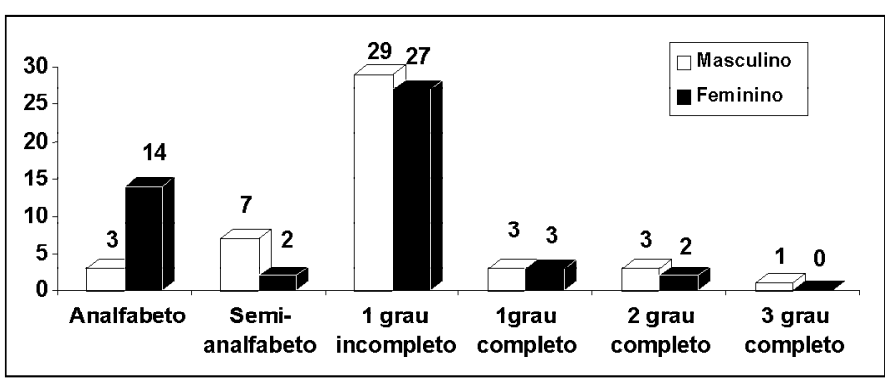

Grafico 1 - Distribuição da amostra total $(n=94)$ por grau de instrução e por sexo.

Os analfabetos, os semi-analfabetos e aqueles com primário incompleto formam o maior número de pacientes entrevistados correspondendo a $87,23 \%$ da amostra (Gráfico 1). Pesquisas realizadas na Região Metropolitana de São Paulo, indicam que esse nível inferior de escolaridade predomina entre as famílias com renda até 4 salários mínimos o que indica a coerência dos resultados da amostra deste trabalho (Pesquisa Origem - Destino (1987) - Metrô de São Paulo). Portanto estes resultados devem ser avaliados apenas como uma tendência, talvez uma amostra maior possa confirmar os valores obtidos.

Um aspecto interessante do perfil da renda dos entrevistados é que $59,57 \%$ têm renda menor do que três salários mínimos (Gráfico 2). Pode-se pensar que um paciente melhor instruído tenha uma renda mais elevada, o que, em nossa amostra, foi confirmado apenas para o grupo CG, no qual foi detectada diferença estatisticamente significante $(p=0,014)$. Este valor pode decorrer do fato deste grupo ser muito pequeno $(n=19)$ ou ainda da grande variância encontrada entre os valores de renda (Tabela 2 ).

O grupo CG tende a apresentar gastos maiores com medicamentos num pós-operatório recente, pois neste período consome grande variedade e quantidade dos mesmos. Entretanto, não se observou diferença estatisticamente significante entre os grupos CG e NC. Este fato deve-se talvez à variância entre os valores.

Não se observou também correlação significativa entre renda familiar e gasto total e gasto com medicamento, nem entre idade e gasto total e gasto com medicamento, fato também observado por Kobelt-Nguyen e col. ${ }^{12}$, levantando outra questão sobre o custo do tratamento do glaucoma.

Uma boa parte da amostra é representada por pacientes aposentados $(48,94 \%)$ e acima de 50 anos $(70,21 \%)$, que em sua grande maioria vive de pensão. Desta forma o ônus do tratamento pode estar incidindo muito mais significativamente nesta parcela dos pacientes. A incidência do glaucoma aumenta com a idade ${ }^{13}$, e países em desenvolvimento, como o Brasil, nos quais a população de idosos está aumentando, terão na próxima década números ainda maiores de portadores de glaucoma, o que obriga o Poder Público a considerar desde já a perspectiva do ônus de crescimento do volume de atendimento dos casos. Programas de prevenção apoiados, financeira e institucionalmente pelo Estado serão de fundamental importância, para evitar que a população carente seja

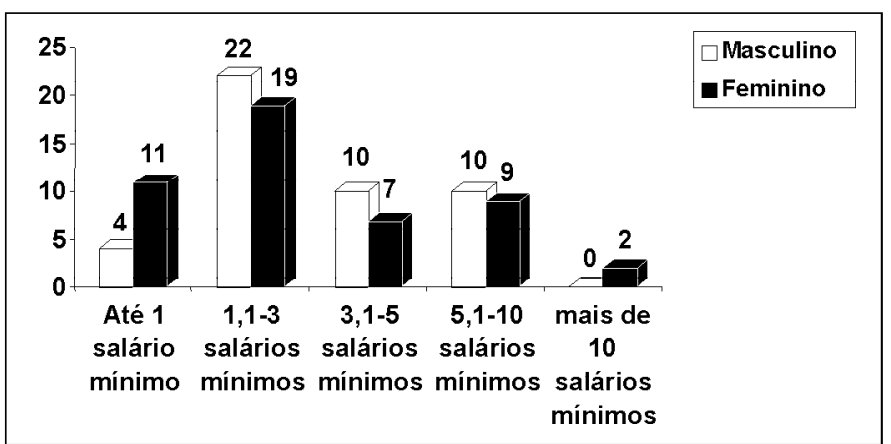

Gráfico 2 - Distribuição da amostra total $(n=94)$ por renda familiar média e por sexo.

levada, por exemplo, a não seguir o tratamento prescrito, particularmente pelo custo do medicamento não importando quão grave possam ser as conseqüências.

A importância deste trabalho foi mostrar o ônus do tratamento na renda familiar de pessoas portadoras de glaucoma, mesmo fazendo uso de um hospital escola previdenciário, e ressaltar que mesmo sem arcarem com custo da consulta médica ou dos exames, o valor do tratamento é bastante elevado. Outros autores já apontam para problemas semelhantes, ainda que não dentro do mesmo assunto. Castro Neto e col., em 1986, já chamavam atenção para o problema da compra dos óculos (paga pelo paciente), em relação à refração (paga pelo governo) ${ }^{14}$. Deve-se, assim, equacionar maneiras de se instruir o paciente quanto ao uso e conservação adequados do medicamento, para que economize o mesmo, e limitar seus retornos ambulatoriais para evitar excesso de visitas.

Cabe observar que os valores monetários estão em reais, na base de abril/97, época da pesquisa, não tendo sido aplicado, qualquer fator de atualização. Este fato, entretanto, não prejudica o objetivo final do trabalho em apresentar o custo relativo do tratamento de glaucoma sobre a renda familiar do paciente, que se mostrou atendido pela coerência estatística.

A redução do número de retornos sem detrimento da qualidade do tratamento é ainda um desafio para o nosso serviço.

\section{SUMMARY}

Purpose: To evaluate the cost of glancoma treatment for an outpatient in a public university hospital.

Patients and Methods: Ninety-four outpatients were randomly Glaucoma Clinic of the Ophthalmology Department of the Universidade Federal de São Paulo, submitted to questionnaires at the regarding personal and antiglaucoma medicine expenses. Data collected, tabulated and the sample was divided into two were groups: surgical and nonsurgical.

Results: The median estimated total spent annually on glaucoma treatment was $R \$ 480.15$, equivalent to $13 \%$ of the median annual family income of the patients. When the sample was divided into two groups, the figures were quite different. The surgical group had a median annual 
estimated total expense of $R \$ 667.94$, equivalent to $19 \%$ of the median annual family income, whereas the nonsurgical group showed a median annual estimated total expense of $R \$ 393.54$ or $11 \%$ of the median annual family income. Sta-tistical difference was found between both groups for total annual cost $(p=0.012)$.

Conclusion: It was observed that the cost of glaucoma treatment is quite high in respect to the family income, even considering the use of a public hospital.

Keywords: Glaucoma, treatment, cost.

\section{REFERÊNCIAS BIBLIOGRÁFICAS}

1. Wilson MR, Martone JF. Epidemilogy of chronic open angle glaucoma. In: Ritch R, Shields MB, Krupin T: The glaucomas. St Louis Mosby $3^{\text {rd }}$ ed. 1996 Vol I;20:407-45.

2. Elkington AR, Lewry J, MacKean J, Sargent P. A collaborative hospital glaucoma survey. Res Clin Forums 1982;4:31-41.

3. Jones SJ, Vernon SA, Cater L, Henry DJ. Costing a community based screening programe for the detection of glaucoma. Eye 1990;4:98-100.

4. Anonymous. Professional news. Optom Manag 1989;25(1):1.
5. Ball ST, Schneider E. Cost of ß-adrenergic receptor blocking agents for ocular hypertension. Arch Ophtalmol 1992;110:654-7.

6. Gelvin JB, Goen TM. Dosage cost analysis in glaucoma management. J Am Optom Assoc 1989;60(10):768-70.

7. Stewart WC, Sine C, Cate E. et al. Daily cost of $\beta$-adrenergic blockers therapy. Arch Ophthalmol 1997;115:853-6.

8. Ainsworth JR, Jay JL. Cost of early trabeculectomy versus conventinal management in primary open angle galucoma. Eye 1991;5:322-8.

9. Abdanur LRA, Paranhos Jr. A, Prata Jr. JA, Mello PAA. Análise dos valores de pressão intra-ocular em indígenas. Rev Bras Oftalmol. In press.

10. Calixto NS. Pressão intra-ocular, curva diária de pressão intra-ocular, rigidez parietal e coeficientes tonográficos (médias de normalidade em diferentes grupos etários). Tese apresentada à Fac. Med. UFMG, para concurso de Livre Docência de Clínica Oftalmológica. Belo Horizonte - MG. 1967.

11. Paranhos FRL, Frange VEC, Lafetá AP. Pressão ocular média em uma instituição de ensino - Fundação Hilton Rocha - MG. Rev Bras Oftalmol 51(6):59-61.

12. Kobelt-Nguyen G, Gerdtham U, Alm A. Costs of treating primary open-angle glaucoma and ocular hypertension: A retrospective, observational two-year chart review of newly diagnosed patients in Sweden and the United States. Journal of Glaucoma 1998;7:95-104.

13. Wilson RS. Epidemiology of glaucoma. In: Epstein DL, Allingham RR, Schuman JS. Chandler and Grant's Glaucoma. Baltimore, Maryland $4^{\text {th }}$ ed 1997;641-7.

14. Castro Neto SM, De Senne FM, Matsui IA, França ST, Tarraf U, José NK. Levantamento do custo de óculos na cidade de Campinas, SP. Rev Bras Oftalmol 1988;67(5):40-5.

\section{Seminário Internacional de Córnea e Lentes de Contato}

\section{4 e 25 de março de 2000 - Gran Meliá São Paulo}

\begin{tabular}{|c|c|c|c|c|c|}
\hline $\begin{array}{l}\text { COMIS } \\
\text { Carlos }\end{array}$ & $\begin{array}{l}\text { ORGANIZADORA } \\
\text { Leite Arieta }\end{array}$ & $\begin{array}{l}\text { PRESIDENTE } \\
\text { CIENTIIFICA }\end{array}$ & DA & COMISSÃO & PALESTRANTES \\
\hline $\begin{array}{l}\text { Milton } \\
\text { Newton } \\
\text { Nilo }\end{array}$ & $\begin{array}{l}\text { Ruiz Alves } \\
\text { Kara José } \\
\text { Holzchuh }\end{array}$ & Newton Kara & José & & $\begin{array}{l}\text { Bruce H. Koffler, Lexington, KY - USA } \\
\text { Oliver H. Dabezies, New Orleans, LO - } \\
\text { Susan M. Stenson, New York, NY - USA } \\
\text { Tomy Starck, San Antonio, TX - USA }\end{array}$ \\
\hline
\end{tabular}

SIMPÓSIOS:

PRESBIOPIA, ASTIGMATISMO
AMINIÓTICA, NOVAS LENTES DE CONTATO, OLHO SECO, LENTES DE CONTATO, MEMBRANA

CURSOS PARALELOS

C-1 - FÓRUM DE LENTES DE CONTATO

Coordenadores: Newton Kara José e Wilson A. Marchi Jr. Coordenadores: Rosane Silvestre de Castro e Eliana

C-2 - ADMINISTRACÃO DO CONSULTÓRIO

Coordenador: Carlos Eduardo Leite Arieta

C-3 - EXCIMER LASER I E II

Excimer Laser I - Coordenadores: Milton Ruiz Alves e

Denise de Vuono Chinzon

Excimer Laser II (complicacões) - Coordenadores:

Hamilton Moreira e Denise de Vuono Chinzon

C-4 - ADAPTACÃO DE LENTES DE CONTATO

ESPE CIAIS

Coordenadores: Adamo Lui Netto e Paulo Ricardo de

Oliveira
C-5 - NOVOS EXAMES AUXILIARES EM

OF T A L MOLO G I A

Matuda

C-6 - AUXILIAR DE OFTALMOLOGIA

Coordenadores: Claudia Assis Lima e Lineu Binotti

C-7 - BIOMICROSCOPIA DO SEGMENTO ANTERIOR

Coordenador: Fernando Oréfice

WET L A B S

WL-1 - REFRACÃO - SUDOP

Coordenadores: Nilo Holzchuh e Marilisa Nano Costa

WL-2 - LENTES DE CONTATO

Coordenador: Nilo Holzchuh

INFORMAC̣̃̃ES : JDE COMUNICAÇ̃̃O E EVENTOS

Alameda Santos, 705 - cj. 56 - 01419-001, São Paulo - SP

Tel. 11- 289-4301 / 251-5273 - Fax. 11- 288-8157

E-mail:_jdecomev@uol.com.br 